\title{
Protective and anti-inflammatory effects of silymarin on paraquat-induced nephrotoxicity in rats
}

\author{
Ali Sharifi-Rigi ${ }^{1}$, Esfandiar Heidarian ${ }^{2 *}$ (]) \\ ${ }^{1}$ Student Research Committee, Shahrekord University of Medical Sciences, Shahrekord, Iran \\ ${ }^{2}$ Clinical Biochemistry Research Center, Basic Health Sciences Institute, Shahrekord University of Medical Sciences, Shahrekord, Iran
}

\section{A R T I CLE I N F O}

Article Type:

Original Article

\section{Article History:}

Received: 19 April 2018

Accepted: 1 December 2018

Keywords:

Silymarin

Paraquat

Kidney injury

Oxidative stress

TNF- $\alpha$

\begin{abstract}
A B S T RAC T
Introduction: Paraquat is a quaternary nitrogen herbicide which induces kidney toxicity due to producing oxidative stress. We have investigated the potential protective effects of silymarin on paraquat-induced renal toxicity.

Methods: Twenty-four male rats were divided into three groups, group 1, control group; group 2, rats that received paraquat only ( $25 \mathrm{mg} / \mathrm{kg}$ b.w./day, po); animals in group 3, was treated with paraquat $(25 \mathrm{mg} / \mathrm{kg}$ b.w./day, po) and silymarin $(50 \mathrm{mg} / \mathrm{kg}$ b.w./day, po). Then, the serum and tissue parameters of the oxidative stress and renal histopathological changes were examined.

Results: In group 2 which received paraquat only, a remarkable increase $(P<0.05)$ was observed in serum creatinine, urea, malondialdehyde (MDA), protein carbonyl, and tumor necrosis factor alpha (TNF- $\alpha)$. Also, there was a significant decrease in renal superoxide dismutase, catalase (CAT), ferric reducing ability of plasma (FRAP) and vitamin C in the second group. Oral administration of silymarin significantly decreased serum urea, creatinine, protein carbonyl, MDA, and TNF- $\alpha$ as well as renal histopathological changes.

Conclusion: The present study suggests that silymarin has anti-inflammatory and nephroprotective effects against nephrotoxicity caused by paraquat.
\end{abstract}

Implication for health policy/practice/research/medical education:

Oral administration of silymarin could ameliorate renal histopathological changes in paraquat-induced nephrotoxicity. Also, it reduced serum urea, creatinine, protein carbonyl, and malondialdehyde. Silymarin has anti-inflammatory, nephro-protective and anti-oxidative stress activities. Hence, it might be useful in patients who were exposed to nephrotoxic agents.

Please cite this paper as: Sharifi-Rigi A, Heidarian E. Protective and anti-inflammatory effects of silymarin on paraquatinduced nephrotoxicity in rats. J Herbmed Pharmacol. 2018;9(1):28-34. doi: 10.15171/jhp.2019.05.

\section{Introduction}

Paraquat is a very toxic herbicide used widely across the globe. Paraquat exerts its herbicide effects through interfering with the electron transfer system and inhibiting the conversion of nicotinamide adenine dinucleotide phosphate (NADP) to NADPH. This herbicide causes toxicity through production of anion superoxide $\left(\mathrm{O}_{2}^{-}\right)$, which can led to the production of other reactive oxygen species (ROS), such as radical hydroxyl $\left(\mathrm{OH}^{-}\right)$and hydrogen peroxide $\left(\mathrm{H}_{2} \mathrm{O}_{2}\right)$ (1). Oxidative stress has key role in the toxicity of paraquat and leads to lipid peroxidation, body cell damage, and apoptosis (2). Paraquat is highly toxic for both humans and animals. It leads to some organ systems damage such as lung, liver, kidney, and Parkinson's disease (PD), as well
(3-5). Paraquat-induced renal toxicity is highly common and reported as the first systemic effects of the paraquat toxicity. A rapid increase in serum creatinine level have been observed in previous reports of paraquat toxicity, which was indicating a decrease in kidneys glomerular filtration rate (GFR). Paraquat causes impaired renal function which is accompanied by reduction of the renal clearance rate and increasing of paraquat level, toxicity, and other organ dysfunctions (6,7). Administration of thioacetamide has been shown to induce liver and kidney injuries and silymarin ameliorated some parameters such as DNA fragmentation, nitric oxide, oxidative stress, collagen content, and liver and kidney histopathological changes in thioacetamide-induced toxicity (8). Silymarin is a potent antioxidant that can scavenge free radicals 
and ROS (9). Silymarin is a flavonolignan containing a combination of silibinin, silydianin and silychristin, which is extracted from the seeds of Silybum marianum $(10,11)$. This antioxidant compound is known as a safe and healthy herbal product (12). Silymarin is used in treatment of liver disorders, such as cirrhosis, chronic liver inflammation, and renal disorders $(13,14)$. In this study, we've investigated the protective effects of silymarin on serum urea, creatinine (Cr), malondialdehyde (MDA), renal MDA, catalase (CAT), superoxide dismutase (SOD), vitamin $\mathrm{C}$, and TNF- $\alpha$ gene expression in paraquatinduced renal toxicity in rats.

\section{Materials and Methods}

Chemicals

Paraquat (200 g/L paraquat dicholoride) was purchased from Shandong Luba Chemical Co. Ltd., Jinan, China. Urea and Cr kits were purchased from Pars Azmoon Co. (Tehran, Iran). Sodium acetate and thiobarbituric acid were provided from Merck (Darmstadt, Germany). Riboflavin, nitro blue tetrazolium, vitamin C were obtained from Sigma-Aldrich Co. (St. Louis, Mo USA). All other chemicals used were analytical grade.

Animal treatment and experimental design

Twenty-four male Wistar rats (10-12 weeks old, 180$220 \mathrm{~g}$ ), were divided into three groups ( $\mathrm{n}=8$ /group). All animals were kept under normal laboratory conditions $\left(22 \pm 2^{\circ} \mathrm{C}, 60 \pm 5 \%\right.$ humidity, and 12:12 light dark cycle). Animals had access to standard rat pellet diet and water. The rats were divided randomly to three groups of 8 each, group 1 (Normal group) was orally given distilled water for 2 weeks. Group 2 (test group) was orally given paraquat only ( $25 \mathrm{mg} / \mathrm{kg}$ body weight/day) by gastric gavage for 2 weeks (15). Group 3 was orally administered paraquat (25 $\mathrm{mg} / \mathrm{kg}$ body weight/day) and orally treated with silymarin (50 mg/kg body weight/day, po) (16) at an interval of 1 hour for 2 weeks.

After 2 weeks, rats were anesthetized with chloroform and blood specimens collected by cardiac puncture method to separate serum and plasma. Also, kidney sample was removed to determine tumor necrosis factor- $\alpha$ (TNF- $\alpha$ ) gene expression, kidney CAT, SOD, and histopathological examinations.

Biochemical analysis

Urea and $\mathrm{Cr}$ were measured by enzymatic method with auto analyzer system (BT3000, Rome, Italy). Serum TNF- $\alpha$ was evaluated by enzyme-linked immune sorbent assay (ELISA) kit, (Bioassay technology laboratory Shanghai, China).

Serum and renal MDA levels were determined as described previously (17). Plasma antioxidant capacity was estimated by ferric reducing ability of plasma (FRAP) method as described previously (17).
Renal CAT activity was measured by previously described method (18). The activity of the renal SOD was determined in the renal tissue by Beauchamp and Fridovich method (19) and total protein was determined by Bradford method (20).

Renal vitamin $\mathrm{C}$ level in the experimental groups was estimated by Stanley and Omaye method (21) and TNF- $\alpha$ gene expression was evaluated by real-time quantitative PCR (RT-qPCR) and the $\Delta \Delta$ CT method as described previously (22). $\beta$-actin was used as internal control for mRNA expression and normalizing data. The serum protein carbonyl level was assessed by Reznick and Parker's spectrophotometric method (23).

Kidney histopathological studies

Kidney sample in each rat fixed with formalin $20 \%$ for histopathological examination. After paraffin embedding, sections prepared at $5 \mu \mathrm{m}$ thickness, stained by hematoxylin-eosin (H\&E) (24), and histological changes were examined by optical microscope.

\section{Statistical analysis}

The results were expressed as mean $\pm \mathrm{SD}$. Analysis of the results was performed using one-way ANOVA by SPSS 20.0 (SPSS Inc., Chicago, IL, USA), and Tukey's post hoc test was used for multiple comparisons. Values of $P<0.05$ were considered significant.

\section{Results}

Effects of silymarin on serum urea, Cr, MDA, plasma FRAP and renal MDA levels

Table 1 shows the effects of paraquat and silymarin on serum urea, Cr, MDA and renal MDA levels in the experimental groups. Administration of paraquat in the second group (group receiving only paraquat) led to a significant elevation $(P<0.05)$ in serum urea, $\mathrm{Cr}$, and MDA and renal MDA levels in comparison with the control group (Table 1). There was a remarkable reduction $(P<0.05)$ in serum urea, $\mathrm{Cr}$, and MDA and renal MDA levels of the group treated with silymarin comparing with the second group $(P<0.05)$.

Group 2 has shown a significant reduction $(P<0.05)$ in FRAP level after receiving paraquat in comparison with the control group (Table 1 ). There was a significant increases $(P<0.05)$ in the plasma FRAP level of group which treated by silymarin when compared with the second group (group receiving only paraquat).

\section{Effects of silymarin on renal CAT and SOD activities}

Figure 1 shows that paraquat significantly decreased $(P<0.05)$ renal CAT and SOD activities compared with the control group. Oral administration of silymarin led to an increase $(P<0.05)$ in renal CAT and SOD activities compared to the second group (group receiving only paraquat). 
Table 1. Effects of silymarin on serum urea, Cr, MDA, plasma FRAP and kidney MDA in paraquat-induced renal injury

\begin{tabular}{llll}
\hline Parameters & Group 1 & Group 2 & Group 3 \\
\hline $\mathrm{Cr}(\mathrm{mg} / \mathrm{dL})$ & $0.42 \pm 0.04$ & $0.66 \pm 0.05^{\mathrm{a}}$ & $0.43 \pm 0.05^{\mathrm{b}}$ \\
Urea $(\mathrm{mg} / \mathrm{dL})$ & $45.37 \pm 3.81$ & $67.50 \pm 2.07^{\mathrm{a}}$ & $55.50 \pm 3.33^{\mathrm{a}, \mathrm{b}}$ \\
FRAP $(\mu \mathrm{M})$ & $518.38 \pm 36.14$ & $393.13 \pm 29.06^{\mathrm{a}}$ & $529.88 \pm 41.22^{\mathrm{b}}$ \\
Serum MDA $(\mu \mathrm{M})$ & $9.99 \pm 1.05$ & $19.95 \pm 1.21^{\mathrm{a}}$ & $11.13 \pm 0.94^{\mathrm{b}}$ \\
Kidney MDA $(\mu \mathrm{mol} / \mathrm{mg}$ protein) & $1.88 \pm 0.03$ & $4.87 \pm 0.03^{\mathrm{a}}$ & $2.50 \pm 0.03^{\mathrm{a}, \mathrm{b}}$ \\
\hline
\end{tabular}

Abbreviatios: FRAP, ferric reducing ability of plasma; MDA, malondialdehyde; $\mathrm{Cr}$, creatinine.

Values are expressed as mean \pm SD. Group 1, normal control; group 2, receiving paraquat only; group 3, rats receiving paraquat and silymarin.

${ }^{a} P<0.05$ compared to group 1.

${ }^{\mathrm{b}} \mathrm{P}<0.05$ compared to group 2 .
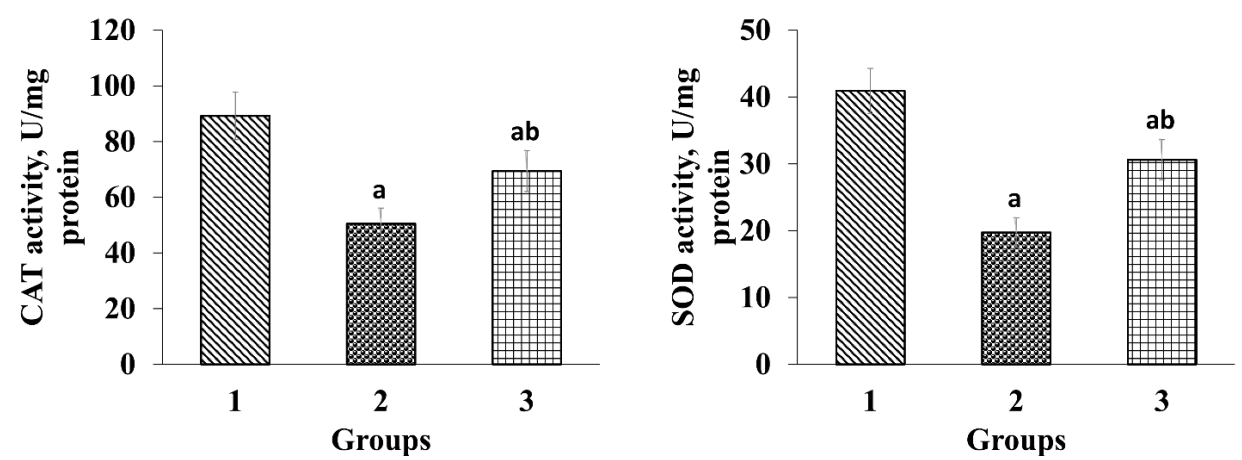

Figure 1. Effects of silymarin on CAT and SOD activities. Values are expressed as mean \pm SD and $n=8$ in each group. Group 1 , normal control; group 2, received paraquat only; group 3 , rats supplemented with paraquat and silymarin.

a $P<0.05$ compared to group 1.

${ }^{\mathrm{b}} P<0.05$ compared to group 2 .
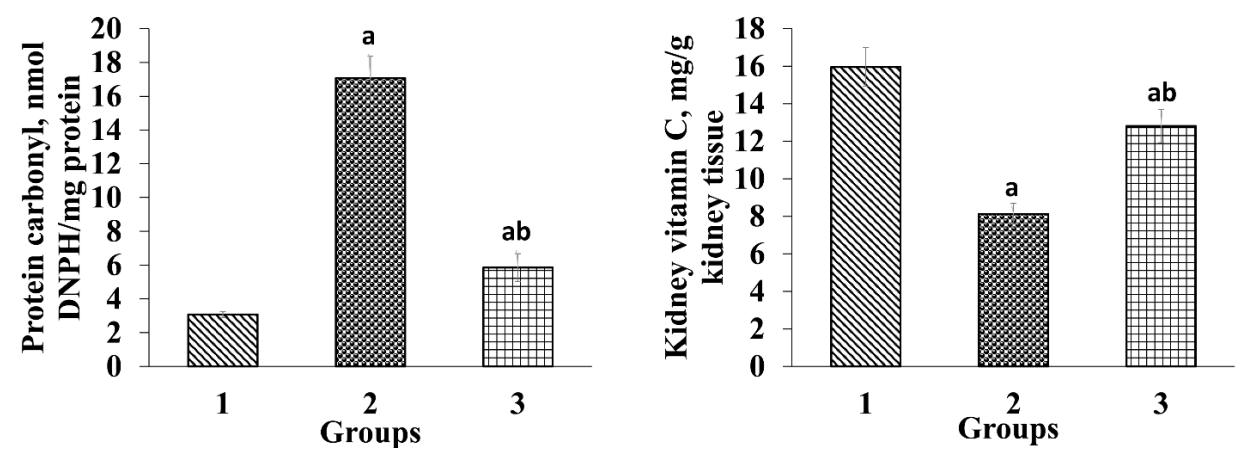

Figure 2. Effects of silymarin on serum PC and vitamin C levels. Values are expressed as mean \pm SD and $n=8$ in each group. Group 1 , normal control; group 2, received paraquat only; group 3, rats supplemented with paraquat and silymarin.

${ }^{\text {a }} P<0.05$ compared to group 1.

${ }^{\mathrm{b}} P<0.05$ compared to group 2 .

Effects of silymarin on the renal vitamin C and PC levels Figure 2 shows the effects of silymarin on the renal vitamin $\mathrm{C}$ and $\mathrm{PC}$ levels. Oral administration of paraquat in the second group led to a significant decrease $(P<0.05)$ in the renal vitamin $\mathrm{C}$ level compared to the control group. On the other hand, administration of silymarin has increased the renal vitamin $C$ level significantly compared to the paraquat group $(P<0.05)$. Also, there was a significant increase $(P<0.05)$ in serum $P C$ levels in rats receiving only paraquat when compared to the control group (Figure 2). Nevertheless, administration of silymarin has significantly reduced $(P<0.05)$ the serum $P C$ level in comparison with the second group.

Effects of silymarin on serum and renal TNF- $\alpha$ gene expression

Figure 3 shows the effects of silymarin on serum and renal TNF- $\alpha$ levels. In the second group (group receiving only 

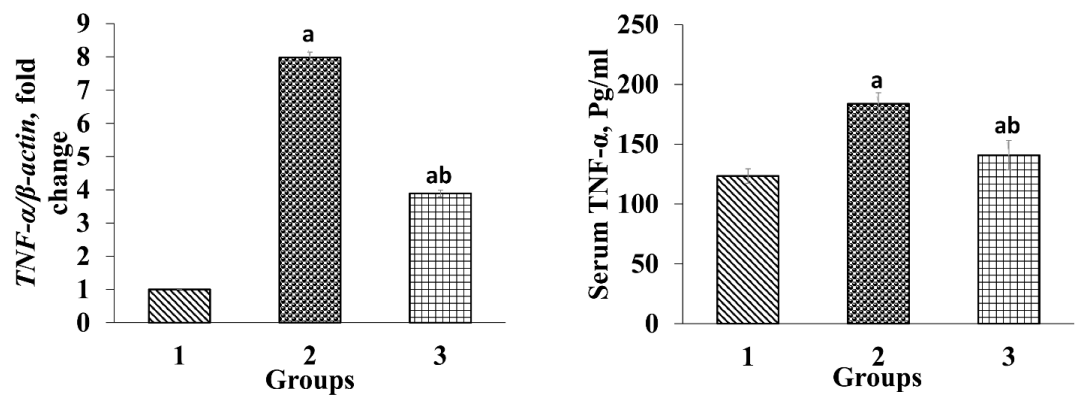

Figure 3. Effects of silymarin on serum TNF- $\alpha$ and expression of $T N F-\alpha$ gene. Values are expressed as mean \pm SD and $n=8$ in each group. Group 1 , normal control; group 2, received paraquat only; group 3, rats supplemented with paraquat and silymarin.

a $P<0.05$ compared to group 1.

${ }^{\mathrm{b}} P<0.05$ compared to group 2 .

paraquat), administration of paraquat led to a significant increase $(P<0.05)$ in serum TNF- $\alpha$ levels and its renal gene expression than the control group. Oral administration of silymarin could significantly decrease $(P<0.05)$ serum and renal TNF- $\alpha$ gene expression compared to the second group (group receiving only paraquat) (Figure 3 ).

\section{Histopathological findings}

Microscopic studies of the second group (group receiving only paraquat) have shown lymphocyte infiltration compared to the control group (Figure 4). In the group treated with silymarin, a significant decrease in lymphocyte infiltration was seen compared to the second group (group receiving only paraquat).

\section{Discussion}

Paraquat is secreted by proximal tubules before renal damage, but tubular damages happen with an increase in paraquat concentration. In paraquat-induced renal toxicity, primarily the glomerular coatings (podocytes) are damaged; then, tubular degeneration, and the formation of eosinophilic granular cytoplasm is happen in distal and proximal tubules (25-27).

Paraquat-induced nephrotoxicity is a common causes of mortality in this group of patients (28). Urea and Cr are among the markers of renal toxicity workup (29). In this study, there is an increase in serum urea and Cr levels in group 2 (Table 1), which indicates the paraquat-induced renal damage; which was consistent with previous studies $(27,29,30)$. In our study, administration of silymarin led to protect kidneys against paraquat toxicity and reduces serum urea and Cr levels (Table 1). According to previous reports, silymarin can reduce urea and $\operatorname{Cr}(10,13,31)$. Therefore, these effects of silymarin on paraquat-induced nephrotoxicity may be produced, at least in part, due to its antioxidant properties.

Paraquat toxicity caused by production of free radicals and oxidative agents (32). There are antioxidant systems against oxidative agents in human body. CAT and SOD are considered as enzymatic antioxidants (33). SOD converts superoxide anion into hydrogen peroxide; and CAT converts hydrogen peroxide into oxygen and water (34). In current study, the SOD and CAT levels were decreased (Figure 1), which is in accordance with previous studies that paraquat shown the ability to decrease the SOD and CAT level in kidney tissue (4). Nevertheless, in the present study silymarin administration led to improve the activity of SOD and CAT (Figure 1) and increase their antioxidant effects which is consistent with a previous study (14). Therefore, these effects of silymarin on renal SOD and CAT in paraquat-induced nephrotoxicity may be due to its antioxidant properties.

Vitamin $C$ is a non-enzymatic antioxidant and can protect body against the oxidant agents. There are reports of low vitamin C levels in paraquat-induced renal toxicity in previous studies (35). Our findings shown a reduction of vitamin $\mathrm{C}$ levels in paraquat-induced renal toxicity, as well (Figure 2). Nevertheless, silymarin could reduce the toxic effects of paraquat and consequently increases vitamin $\mathrm{C}$ levels due to its antioxidant properties. On

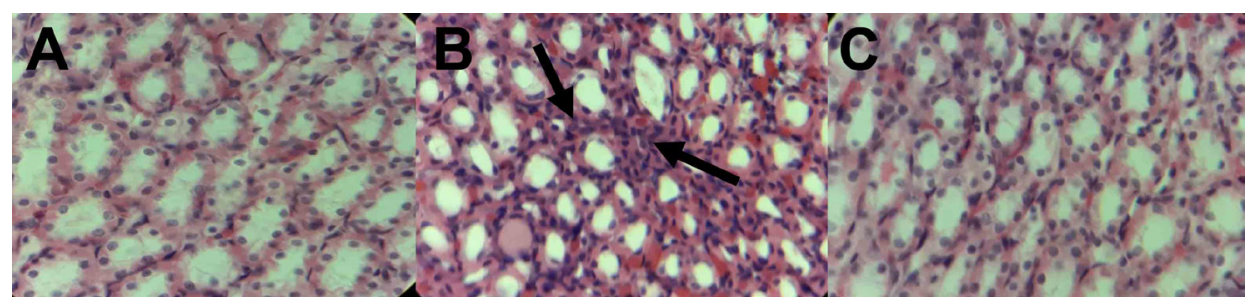

Figure 4. Effects of silymarin on histopathological changes in kidney tissues in rats. A, the normal group (group 1). B, rats treated with paraquat only (group2) showing pathological changes in the kidney such as mononuclear cell infiltration. The black arrows show lymphocyte infiltration. C, (group 3) paraquat-administered rats supplemented with silymarin (50 mg/kg body weight). 
the other hand, paraquat can induce lipid oxidation and protein carbonyl (PC) production through ROS $(36,37)$. MDA is considered as a lipid peroxidation biomarker (38). In the present study, paraquat administration led to increase both the serum and renal MDA and serum protein carbonyl levels by producing oxidant agents which is consist with previous studies $(25,29,35)$. Silymarin administration, however, has reduced serum PC and serum and renal MDA levels (Figure 2), which could be an indication of silymarin effects on inhibiting the oxidation of proteins and lipids, which is consistent with previous results $(9,39)$. Also, our results showed that silymarin have increased the FRAP level (Table 1), which may be due to the silymarin high levels of antioxidant activity, and could justify an increase in SOD and CAT activities and a decrease in MDA and PC by silymarin. In previous studies, an increase in FRAP has been observed by administration of silymarin, as well (40).

Oxidative stress can activate the main mediator of immunity nuclear factor-kappa $\beta(\mathrm{NF}-\kappa \beta)$, which is expressed in excessive inflammation and increases proinflammatory cytokines such as TNF-a, IL-1b, IL-6, and COX-2. It is reported that production of TNF- $\alpha$ in the kidney is responsible for inflammation of the kidney through inducing apoptosis pathway and tubular necrosis $(3,41,42)$. TNF- $\alpha$ is an inflammatory marker and known macrophagic cytokine $(43,44)$. Also, previous studies have shown that paraquat-induced renal toxicity is caused by oxidative stress and inflammation, and ROS plays a role in this toxicity (25). Our findings shown that paraquat increased the serum and renal TNF- $\alpha$ gene expression; which is consistent with previous study (3). Nevertheless, in our study there was a significant decrease in serum and TNF- $\alpha$ gene expression in the silymarin treatment group (Figure 3). Previous studies have also shown that silymarin reduces $\mathrm{TNF} \alpha$ in the presence of renal damage (14). Our histopathologic tests have shown that silymarin reduces infiltration of lymphocytes, apoptosis, and inflammation (Figure 4). Silymarin protects the kidneys against damage and toxicity through reducing the expression of inflammatory factors such as TNF- $a$.

\section{Conclusion}

Silymarin exhibits protective effect against paraquat induced kidney injury. Silymarin increases the level of enzymatic (SOD and CAT) and non-enzymatic (Vit C) antioxidants in the kidney and reduces the expression of inflammatory factors such as TNF-a. Silymarin is considered an appropriate antioxidant against oxidative stress.

\section{Acknowledgment}

We would like to express our gratitude to those who have helped us in Clinical Biochemistry Research Center of Shahrekord University of Medical Sciences.

\section{Authors' contributions}

$\mathrm{EH}$ and ASR contributed in conception, design, data collection, statistical analysis and preparation of the manuscript. EH confirmed the final version of the manuscript for publication.

\section{Conflict of interests}

The authors declare that there is no conflict of interest.

\section{Ethical considerations}

Ethical issues have been observed by the authors. All procedures were approved by the Ethics Committee of Shahrekord University of Medical Sciences, Shahrekord, Iran (Ethic number IR. SKUMS. REC. 1395. 151).

\section{Funding/Support}

This study was funded by Shahrekord University of Medical Sciences (grant no. 2233), Shahrekord, Iran.

\section{References}

1. Atashpour S, Jahromi HK, Jahromi ZK, Zarei S. Antioxidant effects of aqueous extract of Salep on Paraquat-induced rat liver injury. World J Hepatol. 2017;9(4):209-16. doi: 10.4254/wjh.v9.i4.209

2. Li Q, Peng X, Yang H, Wang H, Shu Y. Deficiency of multidrug and toxin extrusion 1 enhances renal accumulation of paraquat and deteriorates kidney injury in mice. Mol Pharm. 2011;8(6):2476-83. doi: 10.1021/ mp200395f

3. Liu Z, Wang X, Wang Y, Zhao M. NLRP3 inflammasome activation regulated by NF- $\mathrm{BB}$ and DAPK contributed to paraquat-induced acute kidney injury. Immunol Res. 2017;65(3):687-98.

4. Tan D, Wang Y, Bai B, Yang X, Han J. Betanin attenuates oxidative stress and inflammatory reaction in kidney of paraquat-treated rat. Food Chem Toxicol. 2015;78:1416.

5. Singh D, Kumar V, Singh C. IFN- $\gamma$ regulates xanthine oxidase-mediated iNOS-independent oxidative stress in maneb-and paraquat-treated rat polymorphonuclear leukocytes. Mol Cell Biochem. 2017;427(1-2):133-43.

6. Mohamed F, Buckley NA, Jayamanne S, Pickering JW, Peake P, Palangasinghe C, et al. Kidney damage biomarkers detect acute kidney injury but only functional markers predict mortality after paraquat ingestion. Toxicol Lett. 2015;237(2):140-50. doi: 10.1016/j.toxlet.2015.06.008.

7. Wunnapuk K, Liu X, Peake P, Gobe G, Endre Z, Grice JE, et al. Renal biomarkers predict nephrotoxicity after paraquat. Toxicol Lett. 2013;222(3):280-8.

8. Ghosh S, Sarkar A, Bhattacharyya S, Sil PC. Silymarin protects mouse liver and kidney from thioacetamide induced toxicity by scavenging reactive oxygen species and activating pi3k-Akt pathway. Front Pharmacol. 2016;7:481. doi: 10.3389/fphar.2016.00481.

9. Oda SS, El-Ashmawy IM. Protective effect of silymarin on mercury-induced acute nephro-hepatotoxicity in 
rats. Global Veterinaria. 2012;9(4):376-83.

10. El-Shitany NA, El-Haggar S, El-Desoky K. Silymarin prevents adriamycin-induced cardiotoxicity and nephrotoxicity in rats. Food Chem Toxicol. 2008;46(7):2422-8. doi: 10.1016/j.fct.2008.03.033.

11. Amien AI, Fahmy SR, Abd-Elgleel FM, Elaskalany SM. Renoprotectiveeffect of mangiferaindica polysaccharides and silymarin against cyclophosphamide toxicity in rats. J Basic Appl Zool. 2015;72:154-62.

12. Karimi G, Vahabzadeh M, Lari P, Rashedinia M, Moshiri M. "Silymarin", a promising pharmacological agent for treatment of diseases. Iran J Basic Med Sci. 2011;14(4):308-17.

13. Karimi G, Ramezani M, Tahoonian Z. Cisplatin nephrotoxicity and protection by milk thistle extract in rats. Evid Based Complement Altern Med. 2005;2(3):383-6.

14. Kabel A, Mahmoud H, El Kholy S. Ameliorative potential of gemfibrozil and silymarin on experimentally induced nephrotoxicity in rats. Afr J Urol. 2013;19(4):171-8.

15. Akinloye $\mathrm{O}$, Abioye $\mathrm{O}$, Olaojoyetan $\mathrm{O}$, Awosika $\mathrm{O}$, Akinloye D. Dose-dependent effects of paraquat on c-reactive protein, some lipid profile parameters and histology of tissues in male albino rats. Ife J Sci. 2013;15(1):189-95. doi: 10.4172/2168-9652.1000106.

16. Kose E, Sapmaz HI, Sarihan E, Vardi N, Turkoz Y, Ekinci N. Beneficial effects of montelukast against methotrexate-induced liver toxicity: a biochemical and histological study. Sci World J. 2012;2012. doi: $10.1100 / 2012 / 987508$.

17. Heidarian E, Soofiniya Y. Hypolipidemic and hypoglycemic effects of aerial part of Cynara scolymus in streptozotocin-induced diabetic rats. J Med Plants Res. 2011;5(13):2717-23.

18. HeidarianE,SaffariJ,Jafari-DehkordiE.Hepatoprotective action of Echinophora platyloba DC leaves against acute toxicity of acetaminophen in rats. J Diet Suppl. 2014;11(1):53-63. doi: 10.3109/19390211.2013.859217.

19. Beauchamp C, Fridovich I. Superoxide dismutase: improved assays and an assay applicable to acrylamide gels. Anal Biochem. 1971;44(1):276-87.

20. Bradford MM. A rapid and sensitive method for the quantitation of microgram quantities of protein utilizing the principle of protein-dye binding. Anal Biochem. 1976;72(1-2):248-54.

21. Omaye ST, Turnbull JD, Sauberlich HE. Selected methods for the determination of ascorbic acid in animal cells, tissues, and fluids. Methods Enzymol. 1979;62:3-11.

22. Sharifi-Rigi A, Heidarian E, Amini SA. Protective and anti-inflammatory effects of hydroalcoholic leaf extract of Origanum vulgare on oxidative stress, TNF- $\alpha$ gene expression and liver histological changes in paraquatinduced hepatotoxicity in rats. Arch Physiol Biochem. 2018:1-8. doi: 10.1080/13813455.2018.1437186.

23. Reznick AZ, Packer L. Oxidative damage to proteins: Spectrophotometric method for carbonyl assay. Methods Enzymol. 1994;233:357-63.
24. Carleton HM, Drury RAB, Wallington EA. Carleton's histological technique: Oxford University Press, USA; 1980.

25. Wei T, Tian W, Liu F, Xie G. Protective effects of exogenous $\beta$-hydroxybutyrate on paraquat toxicity in rat kidney. Biochem Biophys Res Commun. 2014;447(4):666-71. doi: 10.1016/j.bbrc.2014.04.074.

26. Yoon SP, Han MS, Kim JW, Chang IY, Kim HL, Chung $\mathrm{JH}$, et al. Protective effects of chitosan oligosaccharide on paraquat-induced nephrotoxicity in rats. Food Chem Toxicol. 2011;49(8):1828-33.

27. Gu S-Y, Yeh T-Y, Lin S-Y, Peng F-C. Unfractionated bone marrow cells attenuate paraquat-induced glomerular injury and acute renal failure by modulating the inflammatory response. Sci Rep. 2016;6:23287. doi: $10.1038 /$ srep23287.

28. Xu J-j, Zhen J-t, Tang L, Lin Q-m. Intravenous injection of Xuebijing attenuates acute kidney injury in rats with paraquat intoxication. World J Emerg Med. 2017;8(1):614.

29. Gao L, Yang S, Liu J, Liu L. Preventive effects of 5-hydroxy-1-methylhydantoin on paraquat-induced nephrotoxicity in rat. Zhonghua Wei Zhong Bing Ji Jiu Yi Xue. 2015;27(4):246-9.

30. Sener G, Sehirli AÖ, Altunbas HZ, Ersoy Y, Paskaloglu $\mathrm{K}$, Arbak S, et al. Melatonin protects against gentamicin-induced nephrotoxicity in rats. J Pineal Res. 2002;32(4):231-6.

31. Ustyol L, Demirören K, Kandemir I, Erten R, Bulan K, Kaba S, et al. Comparative nephroprotective effects of silymarin, $\mathrm{N}$-acetylcysteine, and thymoquinone against carbon tetrachloride-induced nephrotoxicity in rats. Iran Red Crescent Med J. 2017;19(1). doi: 10.5812/ ircmj.37746.

32. Pourahmad J, Hosseini M-J, Bakan S, Ghazi-Khansari M. Hepatoprotective activity of angiotensin-converting enzyme (ACE) inhibitors, captopril and enalapril, against paraquat toxicity. Pestic Biochem Physiol. 2011;99(1):105-10.

33. Wang S, Guo W, Ren J. Stress signaling in paraquatinduced target organ toxicity. React Oxyg Species. 2016;1(2):131-40.

34. Han J, Zhang Z, Yang S, Wang J, Yang X, Tan D. Betanin attenuates paraquat-induced liver toxicity through a mitochondrial pathway. Food Chem Toxicol. 2014;70:100-6. doi: 10.1016/j.fct.2014.04.038.

35. Valipour P, Heidarian E, Khoshdel A, Gholami-Arjenaki M. Protective effects of hydroalcoholic extract of Ferulago angulata against gentamicin-induced nephrotoxicity in rats. Iran J Kidney Dis. 2016;10(4):189-96.

36. de Oliveira MR, Ferreira GC, Schuck PF. Protective effect of carnosic acid against paraquat-induced redox impairment and mitochondrial dysfunction in $\mathrm{SH}$ SY5Y cells: Role for PI3K/Akt/Nrf2 pathway. Toxicol in Vitro. 2016;32:41-54.

37. Doran ML, Knee JM, Wang N, Rzezniczak TZ, Parkes TL, Li L, et al. Metabolomic analysis of oxidative 
stress: Superoxide dismutase mutation and paraquat induced stress in Drosophila melanogaster. Free Radic Biol Med. 2017;113:323-34. doi: 10.1016/j. freeradbiomed.2017.10.011.

38. Agrawal N, Singh N. Correlation of body fat distribution with presence of oxidative stress in obesity. 2017;4(23):1328-30. doi: 10.1007/s12199-013-0341-y.

39. Turgut F, Bayrak O, Catal F, Bayrak R, Atmaca AF, Koc A, et al. Antioxidant and protective effects of silymarin on ischemia and reperfusion injury in the kidney tissues of rats. Int Urol Nephrol. 2008;40(2):453-60. doi: 10.1007/ s11255-008-9365-4.

40. Ghosh S, Sarkar A, Bhattacharyya S, Sil PC. Silymarin protects mouse liver and kidney from thioacetamide induced toxicity by scavenging reactive oxygen species and activating PI3K-Akt pathway. Front Pharmacol. 2016;7:481. doi: 10.3389/fphar.2016.00481.
41. Ocana-Fuentes A, Arranz-Gutierrez E, Senorans F, Reglero G. Supercritical fluid extraction of oregano (Origanum vulgare) essentials oils: anti-inflammatory properties based on cytokine response on THP-1 macrophages. Food Chem Toxicol. 2010;48(6):1568-75.

42. Tracey KJ. The inflammatory reflex. Nature. 2002;420(6917):853-9.

43. Bautista L, Vera L, Arenas I, Gamarra G. Independent association between inflammatory markers (C-reactive protein, interleukin-6, and TNF- $\alpha$ ) and essential hypertension. J Hum Hypertens. 2005;19(2):149-54.

44. Gratacos J, Collado A, Filella X, Sanmarti R, Canete J, Llena J, et al. Serum cytokines (IL-6, TNF- $\alpha$, IL- $1 \beta$ and IFN- $\gamma$ ) in ankylosing spondylitis: a close correlation between serum IL-6 and disease activity and severity. Rheumatol. 1994;33(10):927-31. 